\title{
Community cluster of meningococcal disease by Neisseria meningitidis serogroup C in Andalusia, Spain, March to May 2011
}

J M Mayoral Cortés (josem.mayoral.sspa@juntadeandalucia.es) ${ }^{1}$ E Torres Butrón², M García Fernández³, Z Herrador Ortiz4,

S Huarte Osakar ${ }^{3}, \mathbf{R}_{\text {Santos Luque }}^{3}$, E Pérez Morilla ${ }^{1}$, J Guillén Enriquez ${ }^{1}$

1. Regional Ministry of Health of the Government of Andalusia, Seville, Spain

2. Andalusian Health Service, Seville, Spain

3. Provincial Health Office, Regional Ministry of Health, Seville, Spain

4. Programa de Epidemiología Aplicada de campo (PEAC). Instituto de Salud Carlos III, Spain

Mayoral Cortés JM, Torres Butrón E, García Fernández M, Herrador Ortiz Z, Huarte Osakar S, Santos Luque R, Pérez Morilla E, Guillén Enriquez J. Community cluster of meningococcal disease by Neisseria meningitidis serogroup C in Andalusia, Spain, March to May 2011. Euro Surveill. 2012;17(36):pii=20261. Available online:

http://www.eurosurveillance.org/ViewArticle.aspx?Articleld=20261

Article submitted on 25 November 2011 / published on 6 September 2012

Between March and May of 2011, a cluster of three fatal cases of meningococcal sepsis occurred in Andalusia, Spain, in a municipality with a population of around 20,000 inhabitants. The cases were in their mid-teens to early thirties and were notified to the epidemiological surveillance system of Andalusia (Sistema de Vigilancia Epidemiológica de Andalucía, SVEA) during a 68-day period from March through May 2011. All three were infected with the same strain of Neisseria meningitidis serogroup C genosubtype VR1:5-1;VR2:10-8. None of the cases had been previously vaccinated against $N$. meningitidis serogroup C. Antibiotic post-exposure chemoprophylaxis was administered to close contacts of every diagnosed case. Once the cluster was confirmed, the local population was informed through the media about the control measures taken by the health authorities. The vaccination history against $N$. meningitidis serogroup $\mathrm{C}$ of the population under 25 years-old in the municipality was checked. Vaccination was offered to unimmunised individuals younger than 25 years of age and an additional dose of vaccine was offered to those who had been vaccinated between 2000 and 2006 with a vaccination schedule of three doses before the first year of age. No further cases occurred since the beginning of these actions.

\section{Introduction}

Neisseria meningitidis is a pathogenic bacterium that colonises the nasopharynx, in most cases, in an asymptomatic way [1]. In Spain, individuals who are colonised are mainly affected by serogroups B or C, but rarely develop invasive disease. When invasive disease occurs however, it appears in acute form, frequently with clinical manifestations of meningitis or sepsis. Meningococcal disease (MD) shows high overall mortality rates [1]. The highest rates are associated with serogroup C, which affected $25 \%$ of cases in Spain in 2010 [2]. In Spain, MD is the most common cause of bacterial meningitis in children and the second most common cause in adults. Cases of MD usually appear sporadically distributed in the population during winter and spring [2]. Infrequently, there are epidemic outbreaks associated with a certain strain of N. meningitidis, which are grouped in space and time.

MD has been notifiable on clinical suspicion in Spain since 1981 [3]. In the Andalusian epidemiological surveillance system (Sistema de Vigilancia Epidemiológica de Andalucía, SVEA) included in the national centre of epidemiology (Centro Nacional de Epidemiología, CNE), a case of MD has to be urgently notified within 24 hours of diagnostic suspicion [4]. To initiate appropriate preventive actions, early confirmation of diagnosis and the identification of the serogroup are critical. These are routinely performed in the microbiology laboratory of the hospital within 48 hours of the patient's admission, or in the Neisseria reference laboratory of the national microbiology centre (Centro Nacional de Microbiología, CNM) along with the characterisation of the strain and the genosubtype identification [5].

The meningococcal $C$ conjugate vaccine was introduced to the childhood vaccination schedule in Spain in the year 2000, administered free of charge, in three doses at two, four and six months of age. During the same year, immunisation was extended to young people of up to 18 years of age, by administering one vaccine dose. In addition to individual protection, the meningococcal $C$ conjugate vaccine provides group immunity by decreasing the rate of nasopharyngeal carriers of $\mathrm{N}$. meningitidis. This reduces the transmission of the microorganism and is important for the control of the disease $[6,7]$. Nevertheless, different studies have shown that the antibody response decreases in the years after vaccination [8,9] and that administration of an additional dose from the first year of age boosts antibody response [10]. In Spain, this decrease of immunity and meningococcal $C$ conjugate 
vaccine effectiveness, has been observed especially with regards to individuals born from the year 2000 onwards, who were subject to the 2000 vaccination schedule, while the effectiveness of the vaccine remained high in children between five months and 18 years of age who were born before 2000 and received one dose of vaccine [9]. This fact motivated a change of the vaccination schedule in 2006 in Spain, delaying the third dose to 15 months of age.

The meningococcal $C$ conjugate vaccine has lead to a significant reduction of the incidence of MD by N. meningitidis serogroup $C$ in Spain over the past years among vaccinated age groups and, to a lesser extent, among unvaccinated age groups. In the period from 1999 to 2009 this reduction was of $87 \%$ [2]. The incidence rate of MD by serogroup C in 2009 was 0.14 per 100,000 inhabitants in Spain and 0.21 per 100,000 in Andalusia [2]. The vaccination coverage with three doses against $N$. meningitidis serogroup $\mathrm{C}$ in the cohort of individuals born in 2010 in Andalusia is 90\% (unpublished data).

In this paper, we present the description of a cluster of cases due to $N$. meningitidis serogroup $C$ in a municipality of Andalusia (Spain) and the intervention to prevent further cases. The cluster occurred when different strains of $N$. meningitidis serogroup $C$ were circulating in Andalusia, with a predominance of the strain affecting the cases in this cluster.

\section{Methods}

The case definition of an MD case in SVEA, as in the whole CNE, follows the clinical, laboratory and epidemiological criteria laid down by the European Commission [11]. The MD can occur as meningitis and/ or meningococcemia that may quickly progress to purpura fulminans, shock and death. Laboratory confirmation is established by isolation or detection of $N$. meningitidis nucleic acid from a normally sterile site or a petechial aspirate, detection of $N$. meningitidis antigens from the cerebrospinal fluid (CSF) or Gramnegative diplococci in the CSF. The suspicion of the existence of a community outbreak is established with the appearance of three or more confirmed or probable cases of the same serogroup, which take place within a time interval of three months in a defined community level (e.g. municipality, neighbourhood) and in which, in addition, the incidence is over 10 cases per 100,000 inhabitants [12]. The confirmation of the outbreak is done by genosubtyping in the reference laboratory for meningococci of the CNM.

In the event reported here, the population at risk was considered to be residents or people who regularly visited and/or stayed in the municipality where the cluster of cases occurred from 10 days before hospitalisation of the first case in March to the end of May 2011 [5].

For the epidemiological investigation, close contacts were defined as people who lived with a case, people who spent the night in the same room than a case, classmates (e.g. desk mates, playmates) or colleagues (e.g. office mates) with frequent and continuing contact with a case up to 10 days before the case's hospitalisation and healthcare workers who had direct contact without protection (mask) with nasopharyngeal secretions of the case (e.g. during resuscitation and tracheal intubation procedures) [5]. To calculate vaccination coverage, the information about the vaccination status against $N$. meningitidis serogroup $C$ in this municipality among residents born after 2000 was obtained from the computerised database of the Andalusian vaccination program (Programa de Vacunación de Andalucía, PVA). The list of residents born after 2000 in the municipality was obtained from the municipal register of inhabitants.

\section{Results}

\section{Description of the cluster of cases}

Between March and May 2011, three cases of MD serogroup $C$ in two women in their mid-teens to early thirties and a man in this late twenties occurred in a municipality of Andalusia with a population of around 20,000 inhabitants (31\% aged less than 25 years). The attack rate was 14.2 cases per 100,000 inhabitants. None of the cases had been previously vaccinated against $N$. meningitidis serogroup $C$ and no epidemiological links were found. The three cases lived in the same municipality: two in the same neighbourhood and one in another area away from the others. None of them had relevant medical history and none had travelled recently. No underlying risk factors were identified. All three had severe sepsis with sudden onset and were treated in the intensive care units of different hospitals, where they died in the hours following admission. For the first two cases, N. meningitidis serogroup C was isolated in blood specimens and in CSF at the hospital laboratory and for the third case, deoxyribonucleic acid (DNA) of N. meningitidis serogroup $C$ was detected in blood specimens sent to the CNM reference laboratory. In this laboratory, the same strain of $N$. meningitidis serogroup $C$ genosubtype VR1:5-1;VR2:10-8 was subsequently identified in the three cases.

\section{Intervention}

During the first 24 hours after the notification of any case, close contacts within the 10 days before hospitalisation were sought. For all three cases, a total of 56 personal close contacts were identified in the cases' social environment (e.g. family, classmates, work mates and colleagues). All close contacts were offered post-exposure prophylaxis with oral rifampicin or ciprofloxacin, and meningococcal $C$ conjugate vaccine, if applicable, by the local health services.

At the end of May 2011, following the notification of the third case, a review of the computerised database of PVA was started to get updated information on the immunisation status against $N$. meningitidis serogroup 
$\mathrm{C}$ in the cohorts born since 2000 residing in the municipality. The coverage found in all of them exceeded $92 \%$ according to the schedule in force at each moment.

As an exceptional measure, the administration of one meningococcal $C$ conjugate vaccine dose was recommended to all previously unimmunised people who were less than 25 years-old and who resided in or regularly visited the municipality, due to work, studies or family, and an additional dose was administered to children vaccinated with the schedule used between 2000 and 2006 who had not received any dose after the first year of age.

Based on the information provided by published studies on $N$. meningitidis serogroup C carriers in the population [13], vaccination was not recommended to those over 25 years of age, despite the fact that two cases were over this age. People over 25 years-old were nevertheless not excluded if they requested vaccination.

To prevent social alarm and to improve the results of the intervention, the population at risk was actively informed about the existence of the cluster, disease transmission, preventive measures, as well as the place and time for the vaccine administration. At the Regional Ministry of Health, a document with questions and answers on MD was available through a respective website, and the call centre 'Salud Responde' was also used to answer telephone enquiries on a $24 / 7$ basis [14].

Information was also provided by the health services through the local and regional media (television, press and radio). Meetings were held with local authorities, local schools and parents of students, in order to manage the vaccination campaign. In training sessions for the healthcare professionals of the local healthcare centres, MD and MD prevention and control measures were reviewed.

From 10 days before hospitalisation of the first case in March until the end of May 2011, providing that less than 10 days had passed since their last visit, regular visitors to the municipality were offered the vaccine in their respective municipalities of residence, once they had been identified. In the affected municipality, vaccination campaign of the susceptible under 25 years of age with the meningococcal $C$ conjugate vaccine, took place during five days at the end May 2011. Two offices for exclusive consultations and vaccination, which were attended by medical and nursing staff, were available from 9 a.m. to 8 p.m. in the healthcare centre and elsewhere in the municipality.

Vaccination was also offered to children and students younger than 25 years in an overflow room during school hours at several educational centres. All people under 25 years-old who attended these consultations were previously requested, through the information campaign, to bring their immunisation records to determine their immunisation status against $N$. meningitidis serogroup $C$. Those who did not bring their immunisation records and whose information was not retrievable from the PVA vaccination database were considered not immunised. Telephone numbers from local health services were made available to clarify doubts and provide additional information on vaccination.

Up to 9 June 2011, 3,818 people, not previously immunised, were vaccinated with meningococcal C conjugate vaccine, corresponding to $18.1 \%$ of the population of the municipality. The highest proportion of vaccinated $(29.1 \%)$ in the municipality was reached in the $25-39$ years age-group. The reason for this was that part of this age-group had an already high pre-existing vaccination coverage maintained by the PVA starting from the year 2000. Moreover, despite vaccination recommendations targeting individuals under 25 years-old, those over this age requesting vaccination could obtain it.

\section{Discussion}

$N$. meningitidis has a large genetic diversity, with a high rate of recombination and frequent appearance of new subtypes, associated with the virulence of the disease. In France, particularly virulent peaks associated with the isolation of phenotypes $\mathrm{C}: 2 \mathrm{a}: \mathrm{P}_{1} .5,2$ and $\mathrm{C}: 2 \mathrm{a}: \mathrm{P}_{1} .5$ were observed in 1992 and 2003. A Norwegian study [15] that compared the predominant genosubtype and virulence in the period 1985-2002 showed an increase in mortality in 1994-2002 in Norway, associated with circulating $N$. meningitidis serogroup $C$ genosubtypes C: 15: P1.7, 16/ST -32 and C: 2a/ST-11.

In Andalusia, between July 2010 and July 2011, 42 cases of MD serogroup $C$ were reported with an incidence rate for this period of 0.5 per 100,000 inhabitants. The mean age of these cases was 32.3 years (range: 7 months -40 years), with $67 \%$ over 19 years-old. These cases, with the exception of the cluster reported here, were sporadically distributed in the whole region. Of 27 cases with known genosubtype, 23 (85\%) corresponded to the same genosubtype VR1:5-1;VR2:10-8 than that identified in the cluster. Of the 14 cases who were less than 20 years of age notified in this period, for which information on previous vaccination status was available, all but two were vaccinated. For the two cases who were considered unvaccinated, one was five weeks-old and, due to the young age, had not received the complete vaccination against $N$. meningitidis serogroup $C$. The other was seven months-old, from another country than Spain, without any of the first two doses established in our calendar. Six vaccine failures among the 14 cases were documented; in five of these, cases were correctly vaccinated between 2001 and 2004 and had received three doses at two, four and six months of age. The sixth case received a single dose in 2001 when he was 17 years-old. In five of these vaccine failures, the same $N$. meningitidis serogroup $C$ genosubtype VR1:5-1;VR2:10-8 was identified. 
The 42 cases of MD serogroup C in Andalusia between July 2010 and July 2011, involved 15 deaths. Of the 14 deaths for which a responsible strain was known, 12 were attributable to this dominant strain. This high mortality also coincides with an unusual distribution of cases of MD due to $N$. meningitidis serogroup $C$, by age group. This disease occurs mainly among children of less than 16 years of age and young adults aged between 16 and 24 year-old [12,16,17]. However, the age of the cases in the cluster reported here is strikingly higher, as well as in the total of MD cases reported in Andalusia.

An increase of cases of MD in young adults and older people has also been described in other countries [10]. Although vaccine failures due to loss of immunity is a fact already described $[8-10,18]$, none of the cases in the cluster reported here had a history of vaccination, and vaccination failure does not seem to have occurred as no further cases were observed in the municipality where a high vaccination coverage was estimated in the population (about $92 \%$ among those born after 1998).

The relationship between carriers of $N$. meningitidis serogroup $C$ and $M D$ among the population is complex. A low rate of carriers is associated with a low rate of protective immunity. A recent meta-analysis [19] shows that the percentage of asymptomatic carriers is highest among young adults under 20 years of age, decreasing gradually with age. The carrier status usually lasts months and confers some immunity. In a study of carriers during an outbreak with five cases of MD due to $N$. meningitidis serogroup $C$, serogroup $C$ was isolated in only $3.9 \%$ of positive samples of healthy carriers and only one of them belonged to the genosubtype implicated in the outbreak [13]. In another study of carriers of MD during an outbreak due to $N$. meningitidis serogroup $C$ in the Netherlands, it was observed that the prevalence of carriers of the epidemic strain was lower in the affected population than in other populations with low incidence [20].

In recent years in Andalusia, which has a population of about 8.5 million, there is only one precedent of a similar cluster of MD cases due to the same $N$. meningitidis serogroup C strain. This was in $\mathbf{2 0 0 5}$, when four cases in a municipality of 13,164 inhabitants were reported, three of them in the age range of 25 to 80 years. At the time that this cluster was detected, vaccination coverage for those born between 2000 and 2004 exceeded $92 \%$ and, for those born between 1987 and 1999, $78.35 \%$. The study of the prevalence of healthy carriers of $N$. meningitidis in a representative sample of 150 individuals in the age group 20 to 25 years (unpublished data) was $13.3 \%$ and only one of the carriers (5\%) had N. meningitidis serogroup C corresponding to the epidemic strain.

The intervention carried out following the cluster described in this paper was a challenge for health authorities in Andalusia, mainly due to the three deaths and the social alarm caused by such clusters in small populations. Community outbreaks are difficult to control, and the decision of whether and who to vaccinate, is not simple or well argued in the literature $[1,12,21-24]$.

In general, the use of chemoprophylaxis in personal contacts of a case of MD is considered an effective measure because of its double effect: the elimination of $N$. meningitidis in carriers and the reduction in the attack rate in contacts. Regarding the use of the vaccine in outbreaks, there is less agreement about the criteria to be followed to decide who should be vaccinated in the population. As the most frequently affected are those aged between two and 25 years, this is commonly the recommended vaccination group a priori.

For outbreaks of MD, there are significant challenges for the development and implementation of intervention protocols $[1,25]$. First of all, the lack of evidence supporting the use of chemotherapy or vaccination, especially when there is no history of contact with a case, mainly because of inherent difficulties of the study design due to ethical issues. The second reason is the pressure that the media may come to exert in these situations, because the social alarm created can have effects on the measures taken; creating a demand by the population that does not always reflect the scientific criteria for intervention.

The decision for selecting the population to be vaccinated in the event reported here was based, as described, on the fact that rates of healthy carriers are higher in young people, as well as on the measures adopted in previous similar outbreaks, and criteria that took into account the cost-benefit of the intervention.

After the implementation of preventive measures, no new cases of MD serogroup $C$ occurred in the area. Our results support the need to maintain a quality and comprehensive system of epidemiological and microbiological surveillance that detects, besides cases, the presence of strains that may have higher virulence in order to implement fast-acting measures.

Due to the effect that the introduction of the meningococcal C conjugate vaccine in 2000 in the vaccination schedule in children and young adults can have on the epidemiology of MD, further efforts and research are needed to improve and update knowledge on how to respond in situations similar to the cluster reported here. 


\section{Acknowledgments}

We gratefully acknowledge all who contributed with their work to the study and control of the outbreak. Special thanks to the healthcare and public health professionals at Distrito Sanitario Sevilla Norte.

\section{References}

1. European Centre for Disease Prevention and Control (ECDC) Public health management of sporadic cases of invasive meningococcal disease and their contacts. Stockholm: ECDC; 2010. Available from: http://www.ecdc.europa.eu/en/ publications/Publications/1010_GUI_Meningococcal_guidance. pdf

2. Cano Portero R, Garrido Estepa M. Enfermedad meningocócica en España. Análisis de la temporada 2009-2010. [Meningococcal disease in Spain. Analysis of the 2009-2010 season]. Madrid: National Centre of Epidemiology, Institute of Health Carlos III. Boletín epidemiológico Semanal. Week 48. 2011;19(17): 233-46. Spanish. Available from: http://revista. isciii.es/index.php/bes/article/view/423/45019

3. Mateo Ontañón S. La enfermedad meningocócica en España. Cambio en su patrón etiológico como problema de salud emergente. [Meningococcal disease in Spain. Changes in its etiological pattern as an emerging health problem]. Rev Esp Salud Pública 1998: 72(5): 439-42. Spanish. Available from: http://scielo.isciii.es/pdf/resp/v72n5/enfermedad.pdf

4. Orden de 11 de diciembre de 2008 , por la que se modifica la Orden de 19 de diciembre de 1996 por la que se desarrolla el Sistema de Vigilancia Epidemiológica en la Comunidad Autónoma de Andalucía y se establece la relación de enfermedades de declaración obligatoria. [Order of 11 December 2008 amending the Order of 19 December 1996 and establishing the development of the Surveillance System in Andalusia and the list of notifiable diseases]. B0JA. 2009;4:6971.Spanish. Available from:http://www.juntadeandalucia.es/ salud/export/sites/csalud/galerias/documentos/p_4_p_1_ vigilancia_de_la_salud/normativa_vigilacia_salud/vs_17.pdf

5. Red Nacional de Vigilancia Epidemiológica, Sistema de Vigilancia Epidemiológica de Andalucía (SVEA). Protocolo de Vigilancia y Alerta de enfermedad Meningocócica. [Alert and surveillance protocol for meningoccocal disease]. Seville: SVEA. [Accessed: 02 Sep 2012]. Spanish. Available from: http://www.juntadeandalucia.es/salud/export/sites/csalud/ galerias/documentos/p_4_p_1_vigilancia_de_la_salud/p_ MENINGOCOCICA_2011.pdf

6. De Arístegui, J. Vacunaciones en el niño. De la teoría a la práctica. [Vaccinations in children. From theory to practice]. D.L. SE-879-06. Seville: Dirección General del Salud Pública Salud Pública. Consejería de Salud. 2006. Spanish. Available from: http://www.juntadeandalucia.es/salud/absys/ documento/vacunaciones.pdf

7. Centers for Disease Control and Prevention (CDC). Atkinson W, Hamborsky J, McIntyre L, Wolfe S, editors. Epidemiology and Prevention of Vaccine-Preventable Diseases. 10th ed. Washington DC: Public Health Foundation; 2007.

8. Trotter CL, Andrews NJ, Kaczmarski EB, Miller E, Ramsay ME. Effectiveness of meningococcal serogroup $C$ conjugate vaccine 4 years after introduction. Lancet. 2004;364(9431):365-7.

9. Larrauri A, Cano R, García M, Mateo S. Impact and effectiveness of meningococcal $C$ conjugate vaccine following its introduction in Spain. Vaccine. 2005;23(32):4097-100.

10. National Advisory Committee on Immunization (NACl). Meningococcal $C$ conjugate vaccination recommendations for infants. An Advisory Committee Statement (ACS). Can Commun Dis Rep. 2007;33(ACS-11):1-12.

11. Commission decision of 28 April 2008 amending decision 2002/253/EC laying down case definitions for reporting communicable diseases to the Community network under Decision No 2119/98/EC of the European Parliament and of the Council. Official Journal of the European Union. Luxembourg: Publications Office of the European Union. 18.6.2008: L 159/46. Available from: http://eur-lex.europa.eu/LexUriServ/ LexUriServ.do?uri=0J:L:2008:159:0046:0090:EN:PDF

12. Bilukha OO, Rosenstein N; National Center for Infectious Diseases, Centers for Disease Control and Prevention (CDC). Prevention and Control of Meningococcal Disease. Recommendations of the Advisory Committee on Immunization Practices (ACIP). MMWR Recomm Rep. 2005;54(RR-7):1-21.

13. Patrick DM, Champagne S, Goh SH, Arsenault G, Thomas $\mathrm{E}$, Shaw $\mathrm{C}$, et al. Neisseria meningitidis carriage during an outbreak of serogroup C disease. Clin Infect Dis. 2003;37(9):1183-8.
14. Salud responde. [Accessed: 05 Sep 2012]. Spanish. Available from: http://www.juntadeandalucia.es/ salud/sites/csalud/contenidos/Informacion_General/ c_7_c_3_nuevo_marco_de_relacion_con_la_ciudadania/ salud_responde

15. Smith I, Caugant DA, Høiby EA, Wentzel-Larsen T, Halstensen A. High case-fatality rates of meningococcal disease in Western Norway caused by serogroup C strains belonging to both sequence type (ST)-32 and ST-11 complexes, 1985-2002. Epidemiol Infect. 2006;134(6):1195-202.

16. Jackson LA, Schuchat A, Reeves MW, Wenger JD. Serogroup C meningococcal outbreaks in the United States. An emerging threat. JAMA. 1995;273(5):383-9.

17. Brooks RB, Woods CW, Rosenstein NE. Neisseria meningitidis outbreaks in the United States, 1994-2002. Abstracts of the 41st Annual Meeting of the Infectious Diseases Society of America; San Diego, CA; 2003 Oct 9-12. Abstract 289. p. 81-2.

18. Borrow R, Miller E. Long-term protection in children with meningococcal $C$ conjugate vaccination: lessons learned. Expert Rev Vaccines. 2006;5(6):851-7.

19. Christensen H, May M, Bowen L, Hickman M, Trotter CL. Meningococcal carriage by age: a systematic review and metaanalysis. Lancet Infect Dis. 2010;10(12):853-61.

20. Conyn-van Spaendonck MA, Reintjes R, Spanjaard L, van Kregten E, Kraaijeveld AG, Jacobs PH. Meningococcal carriage in relation to an outbreak of invasive disease due to Neisseria meningitidis serogroup C in the Netherlands. J Infect. 1999;39(1):42-8.

21. Control and prevention of serogroup $C$ meningococcal disease: evaluation and management of suspected outbreaks: recommendations of the Advisory Committee on Immunizations Practices (ACIP). MMWR Recomm Rep. 1997;46(RR-5):13-21.

22. Skoczyńska A, Wasko I, Kuch A, Gołębiewska A, Foryś M, Hryniewicz W. Outbreak of invasive meningococcal disease in Goleniów County, north-west Poland, March 2009. Euro Surveill. 2010;15(34):pii=19646. Available from: http://www. eurosurveillance.org/ViewArticle.aspx?Articleld=19646

23. Ferro A, Baldo V, Cinquetti S, Corziali P, Gallo G, Lustro G, et al. Outbreak of serogroup $C$. meningococcal disease in Veneto region, Italy. Euro Surveill. 2008;13(2):pii=8008. Available from: http://www.eurosurveillance.org/ViewArticle. aspx?Articleld $=8008$

24. Hoek M, Hanquet G, Heuberger S, Stefanoff P, Zucs P, Ramsay $M$, et al. A European survey on public health policies for managing cases of meningococcal disease and their contacts. Euro Surveill. 2008;13(10):pii=8060. Available from: http:// www.eurosurveillance.org/ViewArticle.aspx?Articleld $=8060$

25. Begg N. Policies for public health management of meningococcal disease. J Epidemiol Community Health. 1999;53(9):516.. 